\title{
Quantum computing in optical microtraps based on the motional states of neutral atoms
}

\author{
K. Eckert, ${ }^{1}$ J. Mompart, ${ }^{1,2}$ X. X. Yi, ${ }^{1,3}$ J. Schliemann, ${ }^{4}$ D. Bruß, ${ }^{1}$ G. Birkl, ${ }^{5}$ and M. Lewenstein ${ }^{1}$ \\ ${ }^{1}$ Institute of Theoretical Physics, University of Hannover, Appelstrasse 2, D-30167 Hannover, Germany \\ ${ }^{2}$ Departament de Física, Universitat Autònoma de Barcelona, E-08193 Bellaterra, Spain \\ ${ }^{3}$ Institute of Theoretical Physics, Northeast Normal University, Changchun 130024, China \\ ${ }^{4}$ Department of Physics and Astronomy, University of Basel, CH-4056 Basel, Switzerland \\ ${ }^{5}$ Institute of Quantum Optics, University of Hannover, Welfengarten 1, D-30167 Hannover, Germany
}

(Received 17 June 2002; published 23 October 2002)

\begin{abstract}
We investigate quantum computation with neutral atoms in optical microtraps where the qubit is implemented in the motional states of the atoms, i.e., in the two lowest vibrational states of each trap. The quantum gate operation is performed by adiabatically approaching two traps and allowing tunneling and cold collisions to take place. We demonstrate the capability of this scheme to realize a square root of swap gate, and address the problem of double occupation and excitation to other unwanted states. We expand the two-particle wave function in an orthonormal basis and analyze quantum correlations throughout the whole gate process. Fidelity of the gate operation is evaluated as a function of the degree of adiabaticity in moving the traps. Simulations are based on rubidium atoms in state-of-the-art optical microtraps with quantum gate realizations in the few tens of milliseconds duration range.
\end{abstract}

DOI: 10.1103/PhysRevA.66.042317

PACS number(s): 03.67.Lx, 32.80.Pj, 42.50.Vk

\section{INTRODUCTION}

The development of tools to prepare, manipulate and measure the quantum state of a physical system represents one of the great challenges of modern science and, in particular, it is essential for applications in quantum information processing such as quantum computing. At present a few systems have been identified that should permit quantum computation: molecules in the context of NMR [1], ion traps [2], cavity QED with photons and atoms [3], solid-state devices such as quantum dots [4-6], and trapped neutral atoms [7-11]. For Rydberg atoms in high Q cavities, the engineering of entangled states and the implementation of quantum logic have been demonstrated [12], furthermore a quantum gate has been performed between the internal and external degrees of freedom of an ion in a trap [13]. In NMR systems, quantum algorithms on a few qubits, e.g., Shor's factoring algorithm, have been reported [14].

Neutral atoms are promising candidates for quantum computing for at least two reasons: (i) techniques of cooling and trapping atoms are by now very well established [15]; and (ii) they are comparatively less sensitive to decoherence, e.g., interaction with the "classical" environment. Neutral atoms can be stored and manipulated in optical lattices [16], standard dipole traps [17], and microtraps [18-21]. In particular, magnetic [18] and optical microtraps [19-21] offer an interesting perspective for storing and manipulating arrays of atoms with the eventual possibility to scale, parallelize, and miniaturize the atom optics devices needed in quantum information processing. Moreover, optical microtraps can take advantage of the fact that most of the current techniques used in atom optics and laser cooling are based on the optical manipulation of atoms [19]. Many of the requirements for the implementation of quantum computation [22] have been recently demonstrated in optical microtraps containing $\sim 100$ atoms per site [21], e.g., selective addressing of single trap sites, and initializing and reading out of quantum states in each site. In addition, the possibility to store and detect single atoms in optical dipole traps has been reported [17].

With the demonstration of single-qubit gates being straight forward, what remains to be experimentally demonstrated is the capability of these optical microtraps to perform two-qubit quantum gates. The most prominent examples of such gates include the controlled-NOT (CNOT) gate, the phase gate and the $\sqrt{\mathrm{SWAP}}$ gate $[4,23]$. The latter transforms states $|0\rangle|1\rangle$ and $|1\rangle|0\rangle$, written in the computational basis, to maximally entangled states, while leaving $|0\rangle|0\rangle$ and $|1\rangle|1\rangle$ unaffected, in such a way that after the successive application of two $\sqrt{\text { SWAP }}$ gates the states of the qubits are interchanged. Each one of these two-qubit gates, together with arbitrary single-qubit operations, is universal, i.e., allows to perform any quantum algorithm. In practice, the particular two-qubit gate to be implemented will depend on the physical system under consideration.

With respect to neutral atoms, several different physical mechanisms to perform two-qubit gates have been proposed, ranging from cold controlled collisions $[7,8]$ and dipoledipole interactions [9-11] to purely geometric quantum evolution [24]. In the cold collisional case, a two-qubit phase gate was proposed by adiabatically approaching two traps [7] or by instantaneous state-selective switching of the trapping potentials [8]. In both cases the qubit was encoded in some internal degrees of freedom of the atoms, e.g., spin, Zeeman or hyperfine levels. For cold collisions to take place the atoms have to be brought to close distances, such that their quantum statistical nature has to be taken into account. A detailed study of the role of the bosonic or fermionic character of particles in the context of quantum information in atomic waveguide structures has been done by Andersson et al. $[25,26]$.

Here we address the problem of implementing a quantum gate by adiabatically approaching two bosonic atoms, each stored in a different microtrap. In contrast to the proposals mentioned above, we assume the qubit to be implemented in 


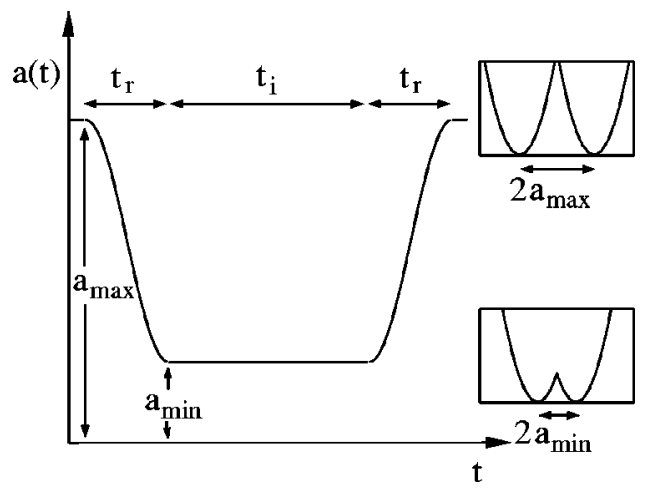

FIG. 1. Separation of the traps as a function of time. $t_{r}$ and $t_{i}$ are the approaching or separating and interaction times, respectively. At $a_{\max }$ atoms located in different traps do not interact, while at $a_{\text {min }}$ tunneling and cold collisions take place.

the motional states of the atoms, i.e., an atom in the ground or the first vibrational state of the trap represents $|0\rangle$ or $|1\rangle$, respectively. Note that, as for the ion-trap case, the observation of neutral atoms cooled down to the ground and first vibrational states as well as superposition states in onedimensional traps has been achieved [27]. To perform the gate operation, we apply the steps outlined in Fig. 1. Initially, the two microtraps are far apart such that the interaction between the two atoms is negligible. Then we adiabatically move both traps close together such that tunneling and cold controlled collisions become important. The dynamics of this process strongly depend on the particular motional state of the atoms and we can make use of this fact to control the interaction such that, after the eventual separation of the traps, the desired gate operation is realized with each trap again containing only one atom.

To be more specific we consider here laser-cooled rubidium atoms stored in optical microtraps [21], assuming that each trap contains initially only one atom. We will show that the $\sqrt{\text { SWAP }}$ gate is the most natural quantum gate to be implemented when the qubit is encoded in the motional states of the atoms and interaction takes place through tunneling and cold collisions. This result applies to both, ${ }^{85} \mathrm{Rb}$ and ${ }^{87} \mathrm{Rb}$, although they have negative and positive scattering length, respectively. In particular, we will demonstrate that a quantum gate of $\sim 20 \mathrm{~ms}$ duration can be performed in state-of-the-art optical microtraps. Very recently, Charron et al. [28] have proposed the implementation of a phase gate in an optical lattice with, as it is done also here, the qubit encoded in the motional states. In this case, a controlled interference set-up was proposed to perform a high-fidelity gate with operation time of $38 \mathrm{~ms}$.

The paper is organized as follows. In Sec. II, we introduce the physical model. Section III is devoted to the implementation of the $\sqrt{\mathrm{SWAP}}$ gate. In Sec. IV we discuss some practical considerations. And, finally, Sec. V summarizes the results and presents the conclusions.

\section{MODEL}

In this section we will first write down the Hamiltonian for the two atoms stored in the microtraps and discuss the interaction mechanism. We will introduce a time-dependent orthonormal set of single-particle states for each trap that is also orthogonal to the states of the other trap for arbitrary distances between the two traps. These single-particle states will make it possible to expand the wave function in a set of two-particle orthonormal states. This representation has two important advantages: (i) it allows to compute entanglement throughout the whole gate process; and (ii) it strongly reduces the computational time required to simulate a quantum gate operation with respect to a direct numerical integration of the Schrödinger equation for the two-particle spatial wave function. Finally, we will discuss the physical implementation of the qubits and its implications for the quantum gate operations.

\section{A. Hamiltonian}

The Hamiltonian governing the dynamics of the two atoms in a time-varying particle-independent trapping potential $V(\vec{r}, t)$ can be written as

$$
H=\sum_{i=1,2}\left[\frac{\vec{p}_{i}^{2}}{2 m}+V\left(\vec{r}_{i}, t\right)\right]+U\left(\vec{r}_{1}-\vec{r}_{2}\right),
$$

where $m$ is the mass of the atoms, $\vec{r}_{i}$ and $\vec{p}_{i}$ are the (threedimensional) position and momentum operators for atoms 1 and 2 , and $U\left(\vec{r}_{1}-\vec{r}_{2}\right)$ accounts for the interaction between the two atoms.

To simplify the problem, we take the trapping potential shape to be time-independent along $y$ and $z$ directions,

$$
V(\vec{r}, t)=v(x, t)+v_{p}(y)+v_{p}(z)
$$

and assume much stronger confinement in $y$ and $z$ directions than in $x$, such that transverse excitations can be neglected. In fact, we will consider that both atoms are cooled down to the $y$ and $z$ vibrational ground states and remain there during all the interaction process. Explicitly, we take the following one-dimensional potential to describe the two microtraps separated by a distance $2 a(t)$ :

$$
v(x, t)=\frac{m \omega_{x}^{2}}{2}\left\{[x+a(t)]^{2} \theta(-x)+[x-a(t)]^{2} \theta(x)\right\},
$$

where $\omega_{x}$ is the trapping frequency in the $x$ direction, and $\theta(x)$ is the step function.

The temporary variation of the trap distance is sketched in Fig. 1. Initially the traps are separated by a distance $2 a_{\max }$. The process of slowly approaching them to a minimum separation $2 a_{\text {min }}$ takes a time $t_{r}$ and is modeled by the first quarter of a period of a cosine. Then we let the atoms interact for a time $t_{i}$ and, finally, we slowly separate the traps.

For cold bosonic atoms, the dominant collisional interaction is due to $s$-wave scattering [8], which can be described by a contact potential of the form

$$
U\left(\vec{r}_{1}-\vec{r}_{2}\right)=\frac{4 \pi a_{t} \hbar^{2}}{m} \delta^{3}\left(\vec{r}_{1}-\vec{r}_{2}\right)
$$


where $a_{t}$ is the $s$-wave scattering length of the rubidium atoms, e.g., in the spin triplet $a_{t}=-369 a_{0}$ for ${ }^{85} \mathrm{Rb}$ and $a_{t}$ $=106 a_{0}$ for ${ }^{87} \mathrm{Rb}$ with $a_{0}$ being the Bohr radius. As long as both atoms remain in the transverse vibrational ground states, we can integrate out the corresponding degrees of freedom and obtain an effective one-dimensional interaction potential [8]

$$
u\left(x_{1}-x_{2}\right)=2 a_{t} \hbar \omega_{p} \delta\left(x_{1}-x_{2}\right),
$$

where $\omega_{p}$ is the transverse trapping frequency. Eqs. (3) and (5) allow us to reduce the complexity of the problem to one dimension.

\section{B. Single-particle states}

We will implement the qubits into the ground and first excited vibrational states of each trap, i.e., we will use the motional states of the atoms. When the two traps are far apart, i.e., $a \alpha \gg 1$ with $1 / \alpha \equiv \sqrt{\hbar / m \omega_{x}}$ being the position uncertainty of the ground state, these states are the energy eigenstates of two displaced one-dimensional harmonic oscillators:

$$
\begin{gathered}
\langle x \mid 0\rangle_{L, R}=\frac{\sqrt{\alpha}}{\pi^{1 / 4}} e^{-\frac{1}{2} \alpha^{2}(x \pm a)^{2}}, \\
\langle x \mid 1\rangle_{L, R}=\frac{\sqrt{2 \alpha}}{\pi^{1 / 4}} e^{-\frac{1}{2} \alpha^{2}(x \pm a)^{2}} \alpha(x \pm a),
\end{gathered}
$$

with $L$ and $R$ labeling the left and right trap, respectively. As we approach the two traps, these single-particle states overlap and are no longer orthogonal. To numerically integrate the Schrödinger equation and to compute entanglement throughout the gate process, we construct an orthonormal single-particle basis for arbitrary distances of the two traps by applying the Gram-Schmidt method (see Appendix A). If we denote these new single-particle states by $|\bar{i}\rangle_{s}$ with $i$ $=0,1,2,3, \ldots$ and $s=L, R$ then it holds ${ }_{s}\langle\bar{i} \mid \bar{j}\rangle_{t}=\delta_{i j} \delta_{s t}$. The four states that for large distances correspond to the two lowest states of each trap read

$$
\begin{aligned}
\langle x \mid \overline{0}\rangle_{L, R} & =\langle x \mid 0\rangle_{L, R} \frac{\xi_{0}^{+}+\xi_{0}^{-}}{2}+\langle x \mid 0\rangle_{R, L} \frac{\xi_{0}^{+}-\xi_{0}^{-}}{2}, \\
\langle x \mid \overline{1}\rangle_{L, R}= & \left(\langle x \mid 1\rangle_{L, R}-\frac{x e^{-a^{2} \alpha^{2}}}{\pi^{1 / 4} \alpha^{3 / 2}}\langle x \mid 0\rangle_{R, L}\right) \frac{\xi_{1}^{+}+\xi_{1}^{-}}{2} \\
& +\left(\langle x \mid 1\rangle_{R, L}-\frac{x e^{-a^{2} \alpha^{2}}}{\pi^{1 / 4} \alpha^{3 / 2}}\langle x \mid 0\rangle_{L, R}\right) \frac{\xi_{1}^{-}-\xi_{1}^{+}}{2},
\end{aligned}
$$

where $\xi_{0}^{ \pm}(a)$ and $\xi_{1}^{ \pm}(a)$ are given in Eqs. (A5). For large separation of the traps, i.e., $a \alpha \gg 1$, we have $\xi_{i}^{+}=\xi_{i}^{-}$for all $i$ and thus the $|\bar{i}\rangle_{L, R}$ become the eigenstates of a single harmonic trap centered at $\mp a$. Notice that the $|\bar{i}\rangle_{L, R}$ states have the following symmetry under parity transformation: $\langle x \mid \bar{i}\rangle_{L, R} \mapsto(-1)^{i}\langle x \mid \bar{i}\rangle_{R, L}$. The general proof is given in Appendix A. This property obviously holds for the $|i\rangle_{L, R}$, and the $|\bar{i}\rangle_{L, R}$ are constructed such that this symmetry is maintained.

Although above we have written only four states, for all simulations using these orthogonalized states we will include all states up to $|\overline{3}\rangle_{L, R}$.

\section{Two-particle states}

Let us motivate the two-particle basis which we will use. On one hand, it must satisfy bosonic statistics, i.e., the basis states have to be symmetric under the permutation of the particles. On the other hand the Hamiltonian of this system is symmetric with respect to parity transformation, i.e., $H(x)$ $=H(-x)$, and therefore does not couple states of opposite parity. For this reason we will introduce basis states with well-defined parity. If for this description we limit ourselves again to the four lowest single-particle states, then the bosonic two-particle sector forms a ten-dimensional Hilbert space. Here, we use the following notation $|\bar{m}(1)\rangle_{s}$ $\otimes|\bar{n}(2)\rangle_{t} \equiv|\bar{m}\rangle_{s}|\bar{n}\rangle_{t}$ with 1 and 2 labeling the atoms and $s, t=L, R$. Thus, the bosonic two-particle basis reads

$$
\begin{gathered}
|00\rangle^{+}=\frac{1}{\sqrt{2}}\left(|\overline{0}\rangle_{L}|\overline{0}\rangle_{R}+|\overline{0}\rangle_{R}|\overline{0}\rangle_{L}\right) \\
|01\rangle^{+}=\frac{1}{2}\left(|\overline{0}\rangle_{L}|\overline{1}\rangle_{R}+|\overline{1}\rangle_{R}|\overline{0}\rangle_{L}-|\overline{0}\rangle_{R}|\overline{1}\rangle_{L}-|\overline{1}\rangle_{L}|\overline{0}\rangle_{R}\right) \\
|11\rangle^{+}=\frac{1}{\sqrt{2}}\left(|\overline{1}\rangle_{L}|\overline{1}\rangle_{R}+|\overline{1}\rangle_{R}|\overline{1}\rangle_{L}\right) \\
|\widetilde{0} 0\rangle^{+}=\frac{1}{\sqrt{2}}\left(|\overline{0}\rangle_{L}|\overline{0}\rangle_{L}+|\overline{0}\rangle_{R}|\overline{0}\rangle_{R}\right) \\
|\widetilde{01}\rangle^{+}=\frac{1}{2}\left(|\overline{0}\rangle_{L}|\overline{1}\rangle_{L}+|\overline{1}\rangle_{L}|\overline{0}\rangle_{L}-|\overline{0}\rangle_{R}|\overline{1}\rangle_{R}-|\overline{1}\rangle_{R}|\overline{0}\rangle_{R}\right)
\end{gathered}
$$

$$
|\widetilde{11}\rangle^{+}=\frac{1}{\sqrt{2}}\left(|\overline{1}\rangle_{L}|\overline{1}\rangle_{L}+|\overline{1}\rangle_{R}|\overline{1}\rangle_{R}\right),
$$

and

$$
|01\rangle^{-}=\frac{1}{2}\left(|\overline{0}\rangle_{L}|\overline{1}\rangle_{R}+|\overline{1}\rangle_{R}|\overline{0}\rangle_{L}+|\overline{0}\rangle_{R}|\overline{1}\rangle_{L}+|\overline{1}\rangle_{L}|\overline{0}\rangle_{R}\right)
$$

$$
|\widetilde{00}\rangle^{-}=\frac{1}{\sqrt{2}}\left(|\overline{0}\rangle_{L}|\overline{0}\rangle_{L}-|\overline{0}\rangle_{R}|\overline{0}\rangle_{R}\right),
$$

$$
|\widetilde{01}\rangle^{-}=\frac{1}{2}\left(|\overline{0}\rangle_{L}|\overline{1}\rangle_{L}+|\overline{1}\rangle_{L}|\overline{0}\rangle_{L}+|\overline{0}\rangle_{R}|\overline{1}\rangle_{R}+|\overline{1}\rangle_{R}|\overline{0}\rangle_{R}\right),
$$




$$
|\widetilde{11}\rangle^{-}=\frac{1}{\sqrt{2}}\left(|\overline{1}\rangle_{L}|\overline{1}\rangle_{L}-|\overline{1}\rangle_{R}|\overline{1}\rangle_{R}\right)
$$

The notation at the left-hand side of Eqs. (8) and (9) means the following: superscripts + or - indicate that the twoparticle state has positive or negative parity, respectively, while the tilde accounts for states where, for $a \alpha \gg 1$, both atoms are in the same trap, i.e., double-occupancy states. It is easy to check the symmetry of these two-particle states under the exchange of the atoms by making use of the parity property of states $|\bar{i}\rangle_{L, R}$ discussed after Eqs. (7).

In addition it is worth to mention that in our simulations we will consider up to eight single-particle states which gives rise to a bosonic two-particle Hilbert space of 36 states (20 states having positive parity from which 10 correspond to double occupancy; and 16 states having negative parity with 10 accounting for double occupancy). Finally note that the fact that we are able to expand the wave function into this finite number of two-particle orthogonal states has also an important advantage with respect to the time needed for the simulation of a gate operation. We have checked the accuracy of the restriction of the simulation to this subspace by comparing the results of the simulations to a direct numerical integration of the Schrödinger equation for the two-particle spatial wave function which is about four orders of magnitude slower.

\section{Physical implementation}

We start from two well-separated traps, each containing one atom. In this situation we can neglect the bosonic nature of the particles and forget about the symmetrization [29]. Only then it is possible to speak about well-defined qubits and we choose to introduce labels $A$ and $B$ for the two qubits by labeling the atom found in the left trap by $A$ and the atom in the right trap by $B$.

With the two traps far apart, single-qubit operations, e.g., a Hadamard gate, can be realized by using two laser pulses in a Raman configuration focused solely on one of the traps. The quantum gate operation between two qubits is much more involved. As we approach the traps, due to tunneling there will be a nonvanishing probability to find both atoms in the same trap. Thus we can no longer distinguish the atoms such that bosonic statistics become important and the qubits are no longer well defined. If, however, we approach and separate the traps in such a way that finally there is again one atom in each of the well-separated traps then we can attribute (new) labels $A$ and $B$ to them in the same way as before.

These considerations suggest the following mapping of the states of the computational basis into the two-particle basis states of Eqs. (8) and (9):

$$
\begin{gathered}
|0\rangle_{A}|0\rangle_{B} \rightarrow|00\rangle^{+}, \\
|0\rangle_{A}|1\rangle_{B} \rightarrow|01\rangle \equiv \frac{1}{\sqrt{2}}\left(|01\rangle^{+}+|01\rangle^{-}\right),
\end{gathered}
$$

$$
\begin{gathered}
|1\rangle_{A}|0\rangle_{B} \rightarrow|10\rangle \equiv \frac{1}{\sqrt{2}}\left(|01\rangle^{+}-|01\rangle^{-}\right), \\
|1\rangle_{A}|1\rangle_{B} \rightarrow|11\rangle^{+} .
\end{gathered}
$$

Note that the two-particle states at the right-hand side of Eq. (10) have a trivial evolution at the trapping frequency (or multiples of it) that can be removed by including this phase in the definition of the single-particle states.

We will take states (10) as the starting set for the gate operation and, after setting the initial state, we will adiabatically realize the gate. In this adiabatic regime, if we start in an energy eigenstate the system will follow this timedependent energy eigenstate during the whole gate process. The only allowed transitions are those corresponding to states that (i) are initially degenerate in energy, and, at short distances, (ii) become coupled via tunneling and/or cold collisions. Therefore, in order to find the most suitable gate to be implemented in this system, we have to identify these resonant couplings.

For this aim we will first discuss the ideal case for which there is no interaction between the atoms, i.e., the case where $a_{t}=0$ in Eq. (5). We then have the following resonant couplings:

$$
\begin{gathered}
|00\rangle^{+} \leftrightarrow|\widetilde{00}\rangle^{+}, \\
|01\rangle \leftrightarrow|\widetilde{01}\rangle \leftrightarrow|10\rangle \leftrightarrow|\widetilde{10}\rangle \leftrightarrow|01\rangle, \\
|11\rangle^{+} \leftrightarrow|\widetilde{11}\rangle^{+},
\end{gathered}
$$

where $|\widetilde{01}\rangle \equiv(1 / \sqrt{2})\left(|\widetilde{01}\rangle^{+}+|\widetilde{01}\rangle^{-}\right) \quad$ and $\quad|\widetilde{10}\rangle \equiv(1 / \sqrt{2})$ $\times\left(|\widetilde{01}\rangle^{+}-|\widetilde{01}\rangle^{-}\right)$. Therefore, there is a non-negligible probability (even if we move the two traps adiabatically) to have both atoms in the same trap after the gate operation. Note that the kinetic and trapping terms of the Hamiltonian do not directly couple $|01\rangle$ with $|10\rangle$ since they are singleparticle Hamiltonians and, therefore, they do not allow for the simultaneously change of the motional states of both atoms. The coupling between $|01\rangle$ and $|10\rangle$ is mediated through the double-occupancy states $|\widetilde{01}\rangle$ and $|\widetilde{10}\rangle$. Clearly, in the noninteracting case, a quantum gate operation always has to face with double occupancy that makes the problem hard to handle.

Figures 2(a) and 2(b) show, for a particular parameter set, the final state of the system after the whole process of approaching and separating the traps as a function of the scattering length. In Fig. 2(a) the initial state is $|01\rangle$ and in (b) $|11\rangle^{+}$. Although the scattering length has a constant value that depends on the atom under consideration, it is used in this plot as a free parameter to illustrate the doubleoccupancy problem. Notice that by changing $\omega_{p}$ it is possible to tune the strength of the effective interaction potential, cf. Eq. (5). Figs. 2(a) and 2(b) clearly show that, for $a_{t}$ $=0$, double occupancy is indeed very important in the final state of the system.

The problem of double occupancy is naturally suppressed when one takes into account the interaction between the at- 


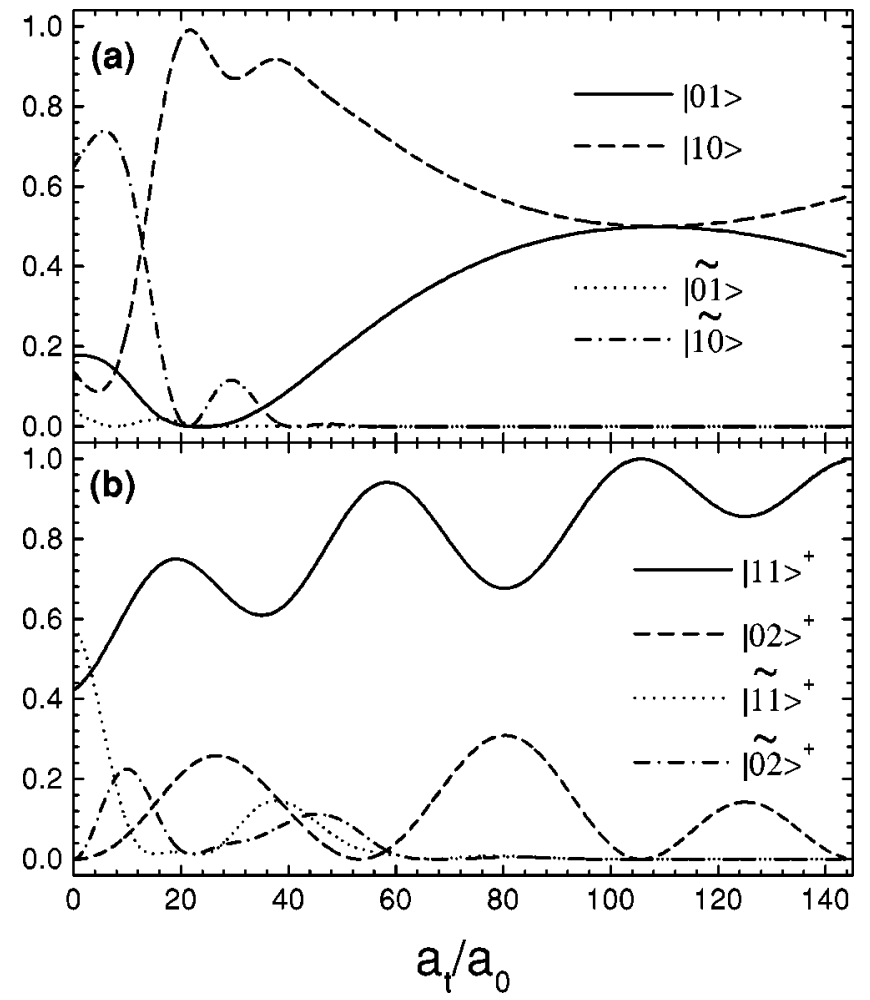

FIG. 2. Populations of the final state of the system after adiabatically approaching and separating the traps as a function of the scattering length. The initial state is (a) $|01\rangle$ and (b) $|11\rangle^{+}$, respectively. The parameter setting is $\omega_{x}=1.25 \times 10^{4} \mathrm{~s}^{-1}, \omega_{p}=4.9$ $\times 10^{5} \mathrm{~s}^{-1}, 1 / \alpha=241 \mathrm{~nm}, a_{\max } \alpha=5, a_{\min } \alpha=1.99, \omega_{x} t_{r}=70$, and $\omega_{x} t_{i}=69$.

oms. In this case, double-occupancy states are no longer degenerate with single-occupancy states and we can neglect the probability to find double occupation in the final state by adiabatically moving the traps. Thus, in the presence of interaction, the resonant couplings read

$$
\begin{gathered}
|01\rangle \leftrightarrow|10\rangle, \\
|11\rangle^{+} \leftrightarrow|02\rangle^{+},
\end{gathered}
$$

where $|02\rangle^{+}=\left(|\overline{0}\rangle_{L}|\overline{2}\rangle_{R}+|\overline{2}\rangle_{R}|\overline{0}\rangle_{L}+|\overline{0}\rangle_{R}|\overline{2}\rangle_{L}+|\overline{2}\rangle_{L}|\overline{0}\rangle_{R}\right) /$ 2. Notice that now the collisional interaction term (5) allows for the simultaneous change of the motional states of both atoms. The role of these couplings is clearly shown in Fig. 2. In Fig. 2(a), where the initial state is $|01\rangle$, double-occupancy populations in the final state start to decrease and eventually vanish as soon as the scattering length is increased. When the initial state is $|11\rangle^{+}$, Fig. 2(b), double occupancy also vanishes as the scattering length increases, but then the population of state $|02\rangle^{+}$becomes important.

Therefore, the coupling given in Eq. (12a) suggests the implementation of a $\sqrt{\text { SWAP }}$ gate, as long as we are able to suppress or control coupling (12b). The degeneracy between $|11\rangle^{+}$and $|02\rangle^{+}$can be broken, for instance, by taking an anharmonic trapping potential such that the vibrational frequencies are no longer equally spaced. In addition, it is possible to adjust the interaction time in such a way that, at the end of the gate operation, state $|02\rangle^{+}$is not populated. In what follows, we will focus on this last possibility.

\section{III. $\sqrt{\text { SWAP }}$ GATE}

The $\sqrt{\mathrm{SWAP}}$ gate has the following effect on the states of the computational basis:

$$
\begin{aligned}
|0\rangle_{A}|0\rangle_{B} \rightarrow|0\rangle_{A}|0\rangle_{B}, \\
|0\rangle_{A}|1\rangle_{B} \rightarrow \frac{1+i}{2}|0\rangle_{A}|1\rangle_{B}+\frac{1-i}{2}|1\rangle_{A}|0\rangle_{B}, \\
|1\rangle_{A}|0\rangle_{B} \rightarrow \frac{1-i}{2}|0\rangle_{A}|1\rangle_{B}+\frac{1+i}{2}|1\rangle_{A}|0\rangle_{B}, \\
|1\rangle_{A}|1\rangle_{B} \rightarrow|1\rangle_{A}|1\rangle_{B} .
\end{aligned}
$$

It is straightforward to check that the successive application of two $\sqrt{\text { SWAP }}$ gates exchanges the states of the qubits, i.e., $U_{\text {SWAP }}=U_{\sqrt{S W A P}} \cdot U_{\sqrt{S W A P}}$. As it has been mentioned before, the $\sqrt{\text { SWAP }}$ gate together with single-qubit operations suffices to realize any quantum algorithm [4] which is not the case for the SWAP gate itself. A simple way to prove this, consists of showing that the universal controlled-NOT gate can be obtained from $\sqrt{\text { SWAP }}$ gates and single-qubit operations. In fact, a possible sequence is (see Appendix B).

$$
U_{\mathrm{CNOT}}=H_{A} \sigma_{A}^{-1} \sigma_{B} U_{\sqrt{\mathrm{SWAP}}} \sigma_{A}^{2} U \sqrt{\mathrm{SWAP}} H_{A}
$$

where $H_{A}$ and $\sigma_{A, B}$ are single-qubit operations. Additionally, sequences involving single-qubit operation exclusively on one of the qubits, e.g., only on A, can be realized [4].

\section{A. Gate simulation}

To simulate the gate operation, we have numerically integrated the time-dependent Schrödinger equation for the Hamiltonian given in Eqs. (3) and (5) with the two-particle wave function expanded in the previously introduced twoparticle basis. Figures 3(a)-3(c) show the result of a $\sqrt{\text { SWAP }}$ gate operation for a scattering length of $a_{t}=106 a_{0}$ corresponding to ${ }^{87} \mathrm{Rb}$ atoms in the spin triplet. The parameter setting is as in Fig. 2 and the initial state is (a) $|00\rangle^{+}$, (b) $|01\rangle$, and (c) $|11\rangle^{+}$. The parameter values are chosen to reproduce the gate operation given in Eq. (13) as well as to suppress the $|02\rangle^{+}$population in the outgoing state of Fig. 3(c). Notice that states representing double occupation are populated at close distances for all three cases. However, these populations vanish after the eventual separation of the traps since the traps are moved adiabatically and singleoccupancy states are not degenerate with double-occupancy ones.

For ${ }^{85} \mathrm{Rb}$ with negative scattering length it is slightly more involved to find parameters for the gate realization since, due to the attractive character of the interaction, double-occupation states can more easily become resonant to single-occupation states, e.g., $|\widetilde{01}\rangle^{+}$with $|00\rangle^{+}$. The parameters must be chosen to avoid this degeneracy between double- and single-occupation states. Fig. 4 shows the result 


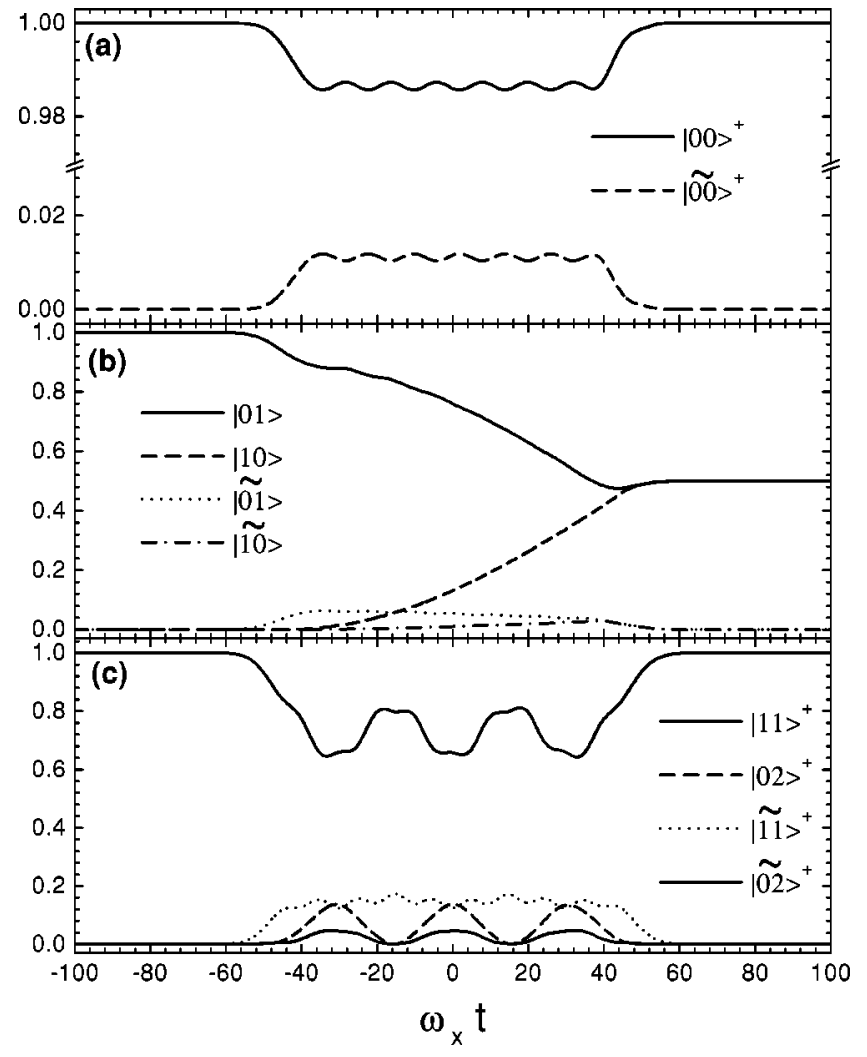

FIG. 3. Simulated $\sqrt{\mathrm{SWAP}}$ gate operation for ${ }^{87} \mathrm{Rb}$ with $a_{t}=106$ $a_{0}$. The rest of parameters as in Fig. 2. The initial state of the system is (a) $|00\rangle^{+}$, (b) $|01\rangle$, and (c) $|11\rangle^{+}$. We note that for (b) the final relative phases of $|01\rangle$ and $|10\rangle$ are as in Eq. (13b).

of a gate simulation for ${ }^{85} \mathrm{Rb}$. Unlike for ${ }^{87} \mathrm{Rb}$, Fig. 4(a) shows that starting from $|00\rangle^{+}$state $|\widetilde{01}\rangle^{+}$is populated during the gate operation.

On the other hand, it is important to notice that the results obtained for ${ }^{87} \mathrm{Rb}$ (Fig. 3) can be also directly implemented in ${ }^{85} \mathrm{Rb}$ by making use of the strong variation of the scattering length in the vicinity of a magnetic-field induced Feschbach resonance [30].

To check the accuracy of the previous simulations in which the two-particle wave function was expanded in a finite set of states, we also have numerically integrated the Schrödinger equation for the two-particle spatial wave function by using an operator split method and an fast Fourier transform (FFT) routine. Figure 5 shows the results of this integration for the same parameter values as in Fig. 3 [31]. The snapshots give the joint-probability distributions for the two particles for three different initial states: (a) $|00\rangle^{+}$, (b) $|01\rangle$, and (c) $|11\rangle^{+}$. The bosonic nature of the atoms manifests in the symmetry of the joint-probability distribution along the diagonal $x_{1}=x_{2}$. In (a) and (c) the final state coincides with the initial one in accordance with Eqs. (13). In (b) $|01\rangle$ evolves towards the maximally entangled state [(1 $+i)|01\rangle+(1-i)|10\rangle] / 2$ whose joint-probability distribution corresponds to the donutlike shape of the last frame.

The accuracy of the simulated gate operation $U$ with respect to the perfect gate operation $U_{\sqrt{S W A P}}$ as given by Eqs. (13) is computed through the averaged fidelity, i.e.,

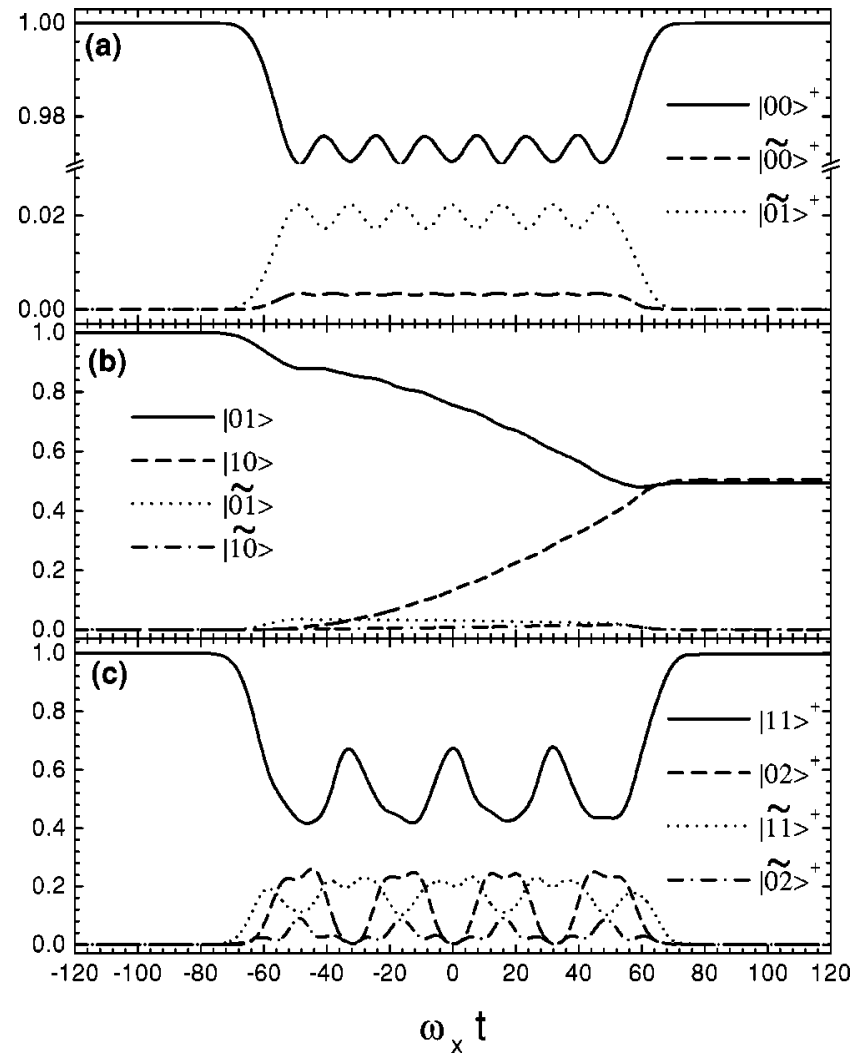

FIG. 4. Simulated $\sqrt{\mathrm{SWAP}}$ gate operation for ${ }^{85} \mathrm{Rb}$ and the following parameter values: $\omega_{x}=1.25 \times 10^{4} \mathrm{~s}^{-1}, \omega_{p}=1 \times 10^{5} \mathrm{~s}^{-1}$, $1 / \alpha=244 \mathrm{~nm}, a_{\max } \alpha=5, a_{\min } \alpha=1.956, \omega_{x} t_{r}=77, \omega_{x} t_{i}=97.2$, and $a_{t}=-369 a_{0}$. The initial state of the system is (a) $|00\rangle^{+}$, (b) $|01\rangle$, and (c) $|11\rangle^{+}$.

$$
F=\overline{\operatorname{Tr}\left[U \rho U^{\dagger} U_{\sqrt{\mathrm{SWAP}}} \rho U_{\sqrt{\mathrm{SWAP}}}^{\dagger}\right]}
$$

where the average is taken over the four orthogonal pure input states $\rho$ from Eqs. (13). Figure 6(a) shows for ${ }^{87} \mathrm{Rb}$ the averaged fidelity $F$ of the gate in the parameter plane $t_{r}$ versus $a_{\text {min }}$. The rest of the parameters are as in Fig. 3. Clearly, the fidelity is very sensitive to the minimum distance due to the exponential dependence of tunneling at this distance. Note that the fidelity of the gate operation corresponding to the parameters of Fig. 3 with a gate duration of $2 t_{r}$ $+t_{i} \sim 17 \mathrm{~ms}$ for $\omega_{x}=1.25 \times 10^{4} \mathrm{~s}^{-1}$ is $F>0.9997$, corresponding to an error rate below $0.1 \%$ per gate operation .

An important issue is how much the gate duration can be decreased while maintaining a high fidelity. In Fig. 6(b) the gate duration is reduced by a factor of 2 which increases the error rate by a factor of 10 . In fact, as soon as the rising time $t_{r}$ is decreased, nonadiabatic effects occur which in turn result in the population of several unwanted states, e.g., double occupation, in the final state of the system. However, it could be possible to use the techniques developed in Ref. [32] to optimize the speed of the gate operation, while suppressing excitations to these unwanted states.

\section{B. Quantum correlations}

Let us consider entanglement in the context of the $\sqrt{\text { SWAP }}$ gate. As already discussed the initial and, as long as double 

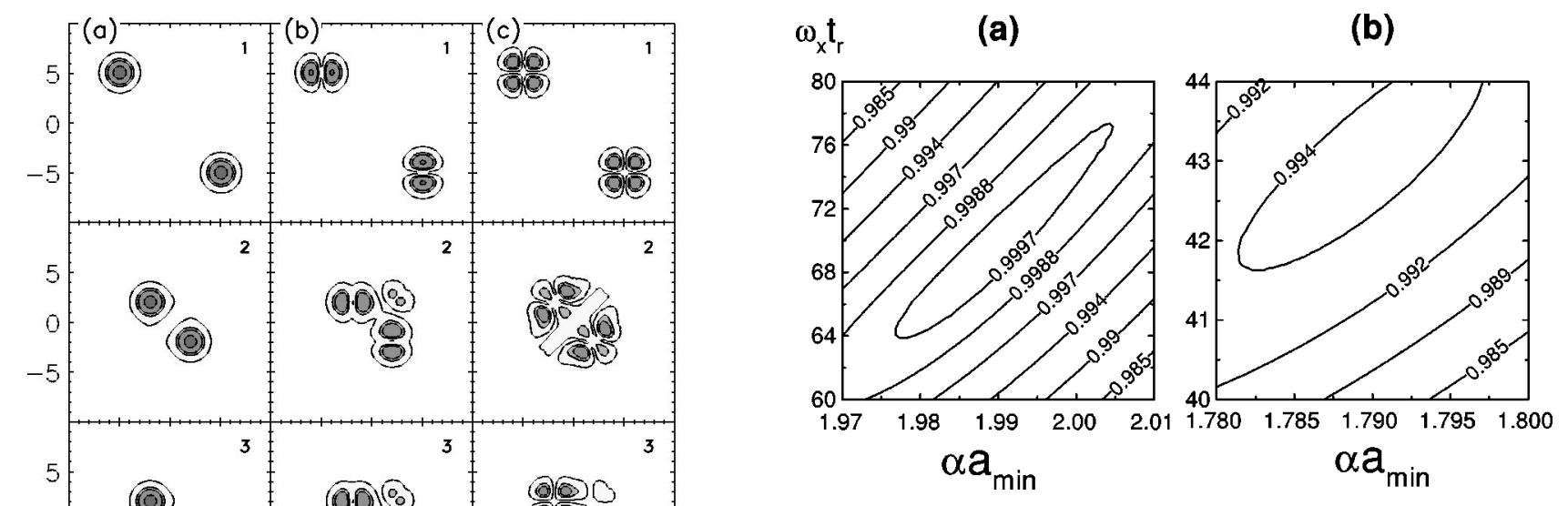

FIG. 6. Averaged fidelity of the gate operation in the parameter plane $t_{r}$ versus $a_{\text {min }}$. The interaction time is (a) $\omega_{x} t_{i}=69$ and (b) $\omega_{x} t_{i}=20$. The rest of the parameters as in Fig. 3 .

occupancy is suppressed, also the final state consist of well separated and thus for practical purposes distinguishable particles, such that the usual notions of entanglement can be used. During the gate operation the particles interact at a short distance and their indistinguishability does not allow to apply the usual concepts of entanglement because we have to distinguish between statistical correlations arising from symmetrization and quantum correlations useful in the context of quantum information. This problem has been discussed in Refs. [6,33] for fermionic two-particle states where the concept of Slater rank and a fermionic correlation measure was derived. In Ref. [6] these methods have been used to study correlations in the context of a quantum gate operation for two electrons in quantum dots. They have been translated to bosons in Ref. [34], and moreover a bosonic von Neumann entropy has been defined in Ref. [35]. Because under the $\sqrt{\text { SWAP }}$ operation the separable state $|01\rangle$ evolves to the maximally entangled state from Eq. (13b), this example does not only provide a good basis to study the creation of entanglement during the process, but it allows also to evaluate to which extend the techniques to analyze quantum correlations of indistinguishable particles can be applied.

Let us write a general pure two-boson state in an $N$-dimensional single-particle space as $|v\rangle$ $=\sum_{i, j=1}^{N} v_{i j} b_{i}^{\dagger} b_{j}^{\dagger}|\Omega\rangle$ where $b_{i}^{\dagger}$ and $b_{i}$ are bosonic creation and annihilation operators and $|\Omega\rangle$ is the vacuum state such that $b_{i}^{\dagger}|\Omega\rangle=|\bar{i}\rangle$. The complex symmetric matrix $v_{i j}=v_{j i}$ is normalized as $\operatorname{tr}\left(v^{\dagger} v\right)=1 / 2$. If new bosonic annihilation operators $b_{i}^{\prime}=\Sigma_{i j} U_{i j} b_{j}$ are introduced by a unitary transformation $U$ of the single particle space, then $v$ transforms as $U v U^{T}$. Now we find that for every symmetric complex matrix $v$ there exists a unitary $U$ such that $U v U^{T}$ is diagonal, i.e., $U v U^{T}=\operatorname{diag}\left[\lambda_{1}, \ldots, \lambda_{r}, 0, \ldots, 0\right]$ with $\lambda_{i}>0[34,35] . r$ is called the Slater rank of $|v\rangle$ and $|v\rangle=\sum_{i=1}^{r} \lambda_{i} b_{i}^{\prime \dagger} b_{i}^{\prime \dagger}|\Omega\rangle$ its Slater decomposition. A bosonic von Neumann entropy can be defined as a function of the Slater coefficients $\lambda_{i}$ [35],

$$
S_{B}=-\sum_{i=1}^{r} \lambda_{k}^{2} \log _{2}\left(\lambda_{k}^{2}\right) \text {. }
$$




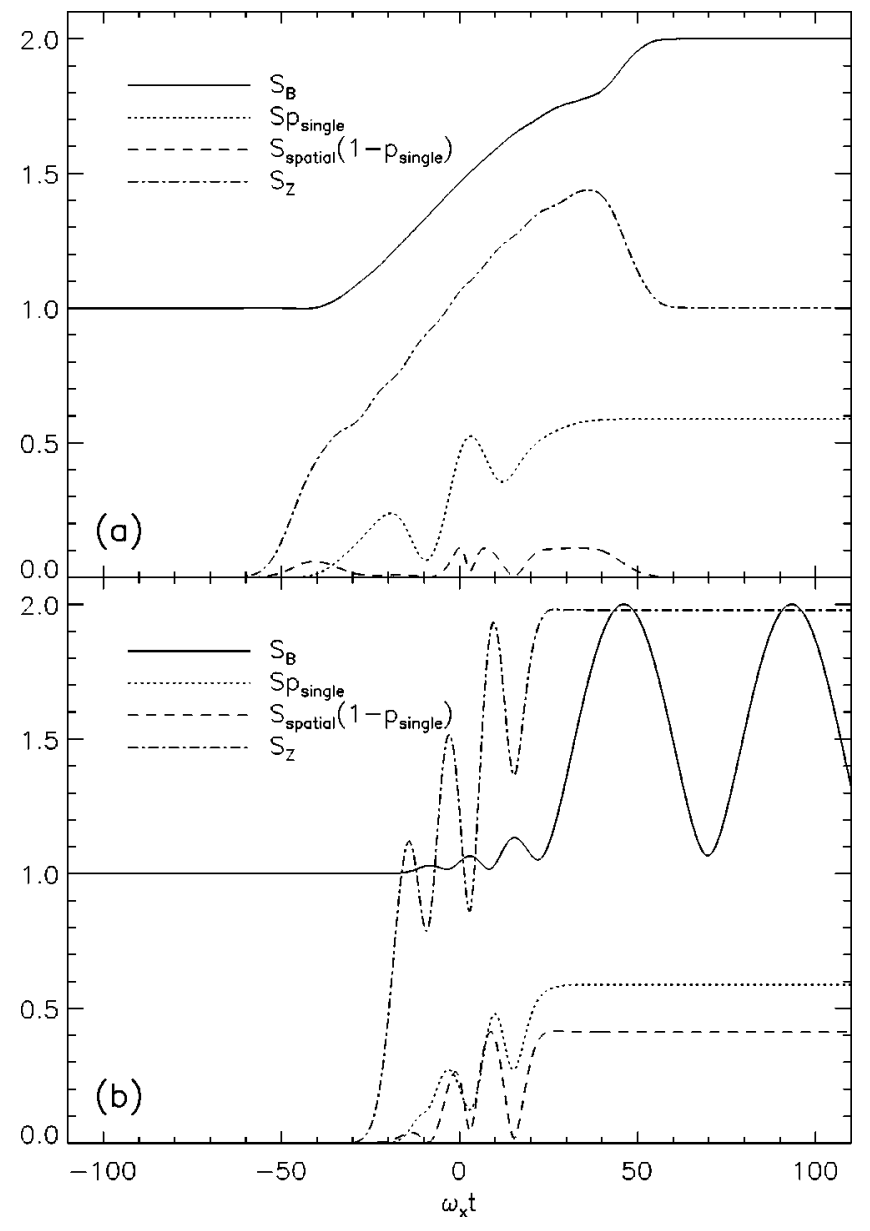

FIG. 7. Quantum correlations (a) for the parameters of Fig. 3, i.e., for the $\sqrt{\text { SWAP }}$ gate operation applied to $|01\rangle$, and (b) for $a_{s}$ $=10 a_{0}, \omega_{x} t_{r}=52.7, \omega_{x} t_{i}=20.4$ and the rest of the parameters as in Fig. 3. The bosonic von Neumann entropy $S_{B}$, the entropy $S p_{\text {single }}$ for particle correlations after projection onto the space spanned by $\left\{|00\rangle^{+},|01\rangle^{+},|01\rangle^{-},|11\rangle^{+}\right\}\left(p_{\text {single }}\right.$ is the probability to find the state in the space spanned by this set), the entropy $S_{\text {spatial }}\left(1-p_{\text {single }}\right)$ for spatial correlations [37], and Zanardi's entropy $S_{Z}$ are plotted.

$S_{B}$ ranges from $S_{B}=0$ for states with Slater rank 1 to $S_{B}$ $=\log _{2}(N)$ for Slater rank $N$ states with all $\lambda_{i}$ equal.

In our case $N=4$ and the initial state $|01\rangle$ has Slater rank two and $S_{B}=1$ while the final state $U_{\sqrt{S W A P}}|01\rangle$ has Slater rank four and $S_{B}=2 . S_{B}(t)$ is plotted in Fig. 7(a) together with $S \cdot p_{\text {single }}$, where $S$ is the von Neumann entropy calculated by projecting onto the space spanned by the set $\left\{|00\rangle^{+},|01\rangle^{+},|01\rangle^{-},|11\rangle^{+}\right\}$and renormalizing. If now states $|\bar{i}\rangle_{L}$ are considered as being distinguishable from states $|\bar{i}\rangle_{R}$ then $S$ can be calculated as for distinguishable particles. $p_{\text {single }}$ is the probability to find the state in the space spanned by the given set. For the $\sqrt{\text { SWAP }}$ gate initially and finally $p_{\text {single }}=1$ holds. We have $S=0$ for $|01\rangle$ and $S=1$ for $U_{\sqrt{S W A P}}|01\rangle$, and the initial and final Schmidt ranks obviously are zero and one.

Although it should be expected that in the limit of large separation the bosonic von Neumann entropy $S_{B}$ and the Slater rank coincide with the von Neumann entropy $S$ and
Schmidt rank, this is clearly not the case here. The reason for this difference is that Slater rank and $S_{B}$ are explicitly constructed as invariants under arbitrary unitary transformations of the complete single-particle space, i.e., these concepts do not distinguish between local and nonlocal transformations [36]. But here it is reasonable to demand invariance only with respect to transformations within a trap.

We already noted in Sec. IID that for certain sets of parameters double occupation in the final state is limiting the fidelity of the gate operation, e.g., for a small value of the scattering length $a_{s}$ [30]. To illustrate that in this case still a large amount of correlations can be present, in Fig. 7(b) we show quantum correlations for a different set of parameters, chosen such that the final state, neglecting symmetrization, reads

$$
c_{10}|1\rangle_{A}|0\rangle_{B}+c_{01}|0\rangle_{A}|1\rangle_{B}+c_{A}|1\rangle_{A}|0\rangle_{A}+c_{B}|0\rangle_{B}|1\rangle_{B},
$$

with $\left|c_{10}\right|^{2}=\left|c_{01}\right|^{2} \sim 0.3$ and $\left|c_{A}\right|^{2}=\left|c_{B}\right|^{2} \sim 0.2$. The first two parts contribute to particle correlations while the last two account for double occupation on site A or B and represent spatial correlations. In Fig. $7(\mathrm{~b})$ the entropy $S_{\text {spatial }}$ corresponding to the latter type of correlations is plotted [37] together with $S$ and $S_{B} . S$ as well as $S_{\text {spatial }}$ are scaled by the probabilities $p_{\text {single }}$ and $1-p_{\text {single }}$, respectively. The oscillations of $S_{B}$ after the traps are eventually separated is due to the interaction that is present if two atoms occupy the same trap. Because $S_{B}$ does not respect locality this interaction can change its value which underlines that in this context correlations are not described by $S_{B}$ in an appropriate way.

In Ref. [38] Zanardi quantifies correlations for indistinguishable particles by mapping bosonic (or fermionic) states to an occupation number basis and calculating the von Neumann entropy $S_{\mathrm{Z}}$ in this basis. This method takes into account the fixed partition of the full single-particle space as well as spatial correlations [36]. If it is applied to the $\sqrt{\text { SWAP }}$ operation then $S_{\mathrm{Z}}=0$ for $|01\rangle$ and $S_{\mathrm{Z}}=1$ for $U_{\sqrt{\mathrm{SWAP}}}|01\rangle$ but in between it has a maximum of $\sim 1.4$. Applied to the operation leading to Eq. (17) we find $S_{\mathrm{Z}}=0$ initially and $S_{\mathrm{Z}}$ $\sim 2$ finally. As can be seen from the above discussion as well as from the analysis in Ref. [36] values $S_{\mathrm{Z}}>1$ are due to mixtures of particle and spatial correlations. It is, however, not clear whether the presence of these different types of correlations leading to $S_{\mathrm{Z}}>1$ can be utilized in this context, where the qubits are explicitly implemented in the vibrational states of each trap.

These results show that for indistinguishable particles interacting at short distances, different types of quantum correlations appear that go beyond the particle correlations explored in the context of the $\sqrt{\mathrm{SWAP}}$ gate. Even if the fidelity is poor, strong quantum correlations can be found in the final state. What remains is to demonstrate their usefulness and to develop a framework that allows to quantify these correlations depending on the implementation of the qubits, on the notion of locality, and on the class of allowed local operations. 


\section{PRACTICAL CONSIDERATIONS}

Scalable systems of optical microtraps based on the dipole force can be realized by focusing a single red-detuned laser beam with a microlens array $[19,21]$. The temporal evolution of the trap separation as shown in Fig. 1 can be realized (i) by using two parallel laser beams focused in such a way that the trapping potentials are longitudinally shifted along the common laser beam direction or (ii) by illuminating the microlens array with two laser beams under slightly different angles with the possible inclusion of an additional twodimensional confining potential perpendicular to the direction of the trap displacement [19]. For the parameters we used in the gate simulations, the minimum distance of $\alpha a_{\min } \sim 2$ corresponds to a separation of the traps of $1 \mu \mathrm{m}$ which is achievable in the present optical microtraps [21]. With laser powers of 1-10 $\mathrm{mW}$ per trap, rubidium atoms can be trapped with typical trapping frequencies along the laser beam direction of $\omega_{x} \sim 10^{4}-10^{5} \mathrm{~s}^{-1}$ while the transverse trapping frequencies can be one or two orders of magnitude larger [19]. Additionally, sideband cooling could be applied to cool the atoms to the ground state of each trap in all dimensions.

In the optical microtrap experiments, the trapping potential is Gaussian shaped with typical depths of $1-10 \mathrm{mK}$ $\times k_{B}$ [19]. For a single trap it is thus a good approximation to assume a harmonic potential for the lower-lying states. For two traps being close together the actual potentials will deviate from the form assumed in Eq. (3). Nevertheless, it is possible to generalize the methods applied here to these particular potentials.

Let us discuss how the error rate of $\$ 1 \%$ arising from nonadiabatic effects as discussed in Sec. III A modifies for this particular implementation. The lifetime of the atoms in the traps is about $100-1000 \mathrm{~ms}$. In this case coherence is mostly limited by spontaneous scattering of photons. Such scattering processes occur in $\sim 10 \mathrm{~ms}$ but as shown in photon echo experiments with strongly confining trapping potentials [39] one atom scatters approximately 50 photons during the coherence time. For the parameters from Fig. 6(b) this gives rise to a qubit error rate of another $2 \%$ such that the total error rate is approximately $3 \%$. If furthermore single-site addressing is desired before and after the operation, then typical initial and final distances between the traps have to be about 5-20 $\mu \mathrm{m}$ which for rubidium means $\alpha a_{\max }=10-40$ instead of $\alpha a_{\max }=5$ which we used in our previous calculations. It is straightforward to estimate that the time needed for the complete process in this case ranges between 18 and $40 \mathrm{~ms}$ with an error rate due to nonadiabatic couplings to other vibrational states of $4-8 \%$. Taking into account the contributions from the spontaneous scattering the cumulated qubit error rate can finally be estimated to lie between 5\% and $12 \%$ which should be enough for proof-of-principle experiments.

In a recent paper, Charron et al. [28] proposed the realization of a phase gate in an optical lattice where the qubits were also implemented in the motional states. Two linear counterpropagating beams from the fundamental and first harmonic of a $\mathrm{CO}_{2}$ laser were used to produce an intensity gradient optical lattice. The barrier between two neighboring traps could be raised or lowered by changing the intensity ratio between the two beams. We notice that the realization of a $\sqrt{\mathrm{SWAP}}$ gate as discussed here should also be possible in this setup although the implementation in optical microtraps presents some advantages such as being not sensitive to the phase fluctuations of the lasers.

\section{CONCLUSION}

We have investigated quantum computation in optical microtraps with the qubits implemented in the motional states of neutral atoms, and tunneling and cold controlled collisions accounting for the interaction between two different qubits. A time-dependent two-particle orthogonal basis has been introduced to simulate the gate operation and to compute entanglement throughout the whole gate process. The bosonic statistic nature of the particles and its role in entanglement has been discussed in detail. We have demonstrated the capability of optical microtraps to realize a high-fidelity $\sqrt{\mathrm{SWAP}}$ gate operation in the few tens of milliseconds range. Finally, some practical considerations for the physical implementation of this quantum gate have been discussed.

\section{ACKNOWLEDGMENTS}

This work was supported by the European Commission through the projects EQUIP and ACQUIRE within the framework of the IST-program, and by the Deutsche Forschungsgemeinschaft through the research program "Quanteninformationsverarbeitung" and SFB 407. J.M. would like to thank the European Community for financial support under Contract No. HPMF-CT-2000-00916. We thank A. Sanpera, W. Ertmer, W. Lange, C. Williams, R. Dumke, F. Hulpke, P. Hyllus, O. Gühne, J. Korbicz, T. Müther, L. Santos, T. Schulte, and M. Volk for helpful discussions.

\section{APPENDIX A: GRAM-SCHMIDT ORTHONORMALIZATION}

In this appendix we will show how to construct the timedependent orthonormal single-particle states, denoted by $|\bar{i}\rangle_{L}$ and $|\bar{i}\rangle_{R}$, from the harmonic oscillator energy eigenstates $|i\rangle_{L}$ and $|i\rangle_{R}$ for the left and the right trap, respectively. We start by defining states involving one state of each trap:

$$
|i\rangle^{ \pm} \equiv \frac{1}{\sqrt{2}}\left[|i\rangle_{L} \pm(-1)^{i}|i\rangle_{R}\right] \quad i=0,1,2,3, \ldots,
$$

where the superscript $+(-)$ indicates positive (negative) parity with respect to the middle between the two traps. We then group these states according to their parity in two sets $S^{ \pm}=\left\{|0\rangle^{ \pm},|1\rangle^{ \pm}, \ldots\right\}$ and focus first on the positive parity set $S^{+}$. This set contains states that are neither orthogonal nor normalized. To perform the orthonormalization, we use the Gram-Schmidt (GM) method starting with the following normalized function: 


$$
\phi_{0}^{+}(x, a) \equiv \frac{\langle x \mid 0\rangle^{+}}{\int\left|\langle x \mid 0\rangle^{+}\right|^{2} d x} .
$$

Then, we define the first linearly independent function $\phi_{1}^{+}$as

$$
\phi_{1}^{+}(x, a)=\frac{\langle x \mid 1\rangle^{+}+a_{10} \phi_{0}^{+}(x, a)}{\int\left|\langle x \mid 1\rangle^{+}+a_{10} \phi_{0}^{+}(x, a)\right|^{2} d x},
$$

where $a_{10}=-\int \phi_{0}^{+}(x, a)\langle x \mid 1\rangle^{+} d x \quad$ which guaranties $\left\langle\phi_{0}^{+} \mid \phi_{1}^{+}\right\rangle=0$. We repeat this procedure to obtain the rest of the linearly independent functions $\phi_{2}^{+}, \phi_{3}^{+}, \ldots$ with positive parity. In an analogous way, we determine from $S^{-}$the set of linearly independent functions $\left\{\phi_{0}^{-}, \phi_{1}^{-}, \phi_{2}^{-}, \phi_{3}^{-}, \ldots\right\}$. An important feature of the GM method when applied to a set of states with the same parity is that the constructed orthonormal states retain the parity of the original set of states. Thus states from $\left\{\phi_{i}^{+}\right\}$and $\left\{\phi_{i}^{+}\right\}$ have positive and negative parity, respectively, and therefore, the whole set $\left\{\phi_{0}^{ \pm}, \phi_{1}^{ \pm}, \phi_{2}^{ \pm}, \phi_{3}^{ \pm}, \ldots\right\}$ is orthonormal. Explicitly, the first four orthonormalized functions read

$$
\begin{gathered}
\phi_{0}^{ \pm}(x, a)=\xi_{0}^{ \pm}(a)\langle x \mid 0\rangle^{ \pm}, \\
\phi_{1}^{ \pm}(x, a)=\xi_{1}^{ \pm}(a)\left(\langle x \mid 1\rangle^{ \pm} \pm \frac{x \alpha^{3 / 2}}{\sqrt[4]{4} \pi} e^{-a^{2} \alpha^{2}}\langle x \mid 0\rangle^{\mp}\right),
\end{gathered}
$$

where

$$
\begin{gathered}
\xi_{0}^{ \pm}(a)=\frac{1}{\sqrt{1 \pm e^{-a^{2} \alpha^{2}}}}, \\
\xi_{1}^{ \pm}(a)=\frac{e^{a^{2} \alpha^{2}}}{\sqrt{\left(e^{a^{2} \alpha^{2}} \pm 1\right)\left(e^{a^{2} \alpha^{2}}-e^{-a^{2} \alpha^{2}} \pm 2 a^{2} \alpha^{2}\right)}} .
\end{gathered}
$$

For the sake of brevity, we do not explicitly show the analytical expressions for the rest of the $\phi_{i}^{ \pm}$.

Once we have obtained the orthonormal set $\left\{\phi_{i}^{ \pm}\right\}$, it is straightforward to write down the single-particle basis that we will use,

$$
\begin{gathered}
\langle x \mid \bar{i}\rangle_{L}=\frac{1}{\sqrt{2}}\left(\phi_{i}^{+}+\phi_{i}^{-}\right), \\
\langle x \mid \bar{i}\rangle_{R}=(-1)^{i} \frac{1}{\sqrt{2}}\left(\phi_{i}^{+}-\phi_{i}^{-}\right),
\end{gathered}
$$

These states are orthonormal due to the orthonormality of the $\phi_{i}^{ \pm}$and in the limit $a \alpha \gg 1$ become the corresponding harmonic oscillator energy eigenstates for each trap. These new orthonormal states do not have in general a well-defined parity with respect to the center of the corresponding trap but it is straightforward to check from Eqs. (A6) that they satisfy the following property under parity transformation with respect to the middle of the traps:

$$
\langle x \mid \bar{i}\rangle_{L, R} \mapsto(-1)^{i}\langle x \mid \bar{i}\rangle_{R, L}
$$

\section{APPENDIX B: UNIVERSALITY OF THE $\sqrt{\text { SWAP GATE }}$}

Our goal here is to write down the sequence of steps required to build the controlled-NOT gate, which, in the computational basis $|0\rangle_{A}|0\rangle_{B},|0\rangle_{A}|1\rangle_{B},|1\rangle_{A}|0\rangle_{B}$, and $|1\rangle_{A}|1\rangle_{B}$, reads

$$
U_{\mathrm{CNOT}}=\left(\begin{array}{llll}
1 & 0 & 0 & 0 \\
0 & 1 & 0 & 0 \\
0 & 0 & 0 & 1 \\
0 & 0 & 1 & 0
\end{array}\right)
$$

from the $\sqrt{\text { SWAP }}$ gate

$$
U_{\sqrt{\mathrm{SWAP}}}=\left(\begin{array}{cccc}
1 & 0 & 0 & 0 \\
0 & \frac{1+i}{2} & \frac{1-i}{2} & 0 \\
0 & \frac{1-i}{2} & \frac{1+i}{2} & 0 \\
0 & 0 & 0 & 1
\end{array}\right) .
$$

The single-qubit operations we need are on one hand the Hadamard gate

$$
H=\frac{1}{\sqrt{2}}\left(\begin{array}{cc}
1 & 1 \\
1 & -1
\end{array}\right),
$$

and on the other hand the following combination of identity and Pauli $\sigma_{z}$ matrices:

$$
\sigma=\left(\begin{array}{cc}
1 & 0 \\
0 & -i
\end{array}\right)=e^{-i \frac{\pi}{4} I} e^{-i \frac{\pi}{2} \sigma_{z}} .
$$

Let us call $H_{A, B}$ and $\sigma_{A, B}$ the corresponding single-qubit operations for qubit $A$ or $B$. Now it is easy to check that the following combination of single-qubit operations and $\sqrt{\mathrm{SWAP}}$ gates yields the phase gate:

$$
U_{P H A S E}=\sigma_{A}^{-1} \sigma_{B} U_{\sqrt{\mathrm{SWAP}}} \sigma_{A}^{2} U_{\sqrt{\mathrm{SWAP}}}=\left(\begin{array}{cccc}
1 & 0 & 0 & 0 \\
0 & 1 & 0 & 0 \\
0 & 0 & 1 & 0 \\
0 & 0 & 0 & -1
\end{array}\right) .
$$

This sequence is not unique, and more sophisticated sequences involving single-qubit operations on only one of the qubits can be implemented [4]. Finally, to obtain the controlled-NOT gate it is enough to apply a Hadamard gate on the qubit $A$ at both sides of Eq. (B5), i.e.,

$$
U_{\mathrm{CNOT}}=H_{A} U_{\mathrm{PHASE}} H_{A} \text {. }
$$


[1] D. G. Cory, A. F. Fahmy, and T. F. Havel, Proc. Natl. Acad. Sci. U.S.A. 94, 1634 (1997); N. A. Gershenfeld and I. L. Chuang, Science 275, 350 (1997); I. L. Chuang, N. Gershenfeld, M. G. Kubinec, and D. W. Leung, Proc. R. Soc. London, Ser. A 454, 447 (1998).

[2] J. I. Cirac and P. Zoller, Phys. Rev. Lett. 74, 4091 (1995); J. I. Cirac and P. Zoller, Nature (London) 404, 579 (2000); T. Calarco, J. I. Cirac, and P. Zoller, Phys. Rev. A 63, 062304 (2001).

[3] A. Barenco, D. Deutsch, A. Ekert, and R. Jozsa, Phys. Rev. Lett. 74, 4083 (1995); T. Sleator and H. Weinfurter, ibid. 74, 4087 (1995); Q. A. Turchette, C. J. Hood, W. Lange, H. Mabuchi, and H. J. Kimble, ibid. 75, 4710 (1995); P. Domokos, J. M. Raimond, M. Brune, and S. Haroche, Phys. Rev. A 52, 3554 (1995); T. Pellizzari, S. A. Gardiner, J. I. Cirac, and P. Zoller, Phys. Rev. Lett. 75, 3788 (1995); L. G. Lutterbach and L. Davidovich, Opt. Express 3, 147 (1998); V. Giovannetti, D. Vitali, P. Tombesi, and A. Ekert, Phys. Rev. A 62, 032306 (2000).

[4] D. Loss and D. P. DiVincenzo, Phys. Rev. A 57, 120 (1998).

[5] G. Burkard, D. Loss, and D. P. DiVincenzo, Phys. Rev. B 59, 2070 (1999); G. Burkard, G. Seelig, and D. Loss, ibid. 62, 2581 (1999).

[6] J. Schliemann, D. Loss, and A. H. MacDonald, Phys. Rev. B 63, 085311 (2001).

[7] D. Jaksch, H. J. Briegel, J. I. Cirac, C. W. Gardiner, and P. Zoller, Phys. Rev. Lett. 82, 1975 (1999).

[8] T. Calarco, E. A. Hinds, D. Jaksch, J. Schmiedmayer, J. I. Cirac, and P. Zoller, Phys. Rev. A 61, 022304 (2000).

[9] G. K. Brennen, C. M. Caves, P. S. Jessen, and I. H. Deutsch, Phys. Rev. Lett. 82, 1060 (1999).

[10] D. Jaksch, J. I. Cirac, P. Zoller, S. L. Rolston, R. Côte, and M. D. Lukin, Phys. Rev. Lett. 85, 2208 (2000).

[11] I. E. Protsenko, G. Reymond, N. Schlosser, and P. Grangier, Phys. Rev. A 65, 052301 (2002).

[12] E. Hagley, X. Maitre, G. Nogues, C. Wunderlich, M. Brune, J. M. Raimona, and S. Haroche, Phys. Rev. Lett. 79, 1 (1997); A. Rauschenbeutel, G. Nogues, S. Osnaghi, P. Bertet, M. Brune, J. M. Raimond, and S. Haroche, ibid. 83, 5166 (1999); J. M. Raimond, M. Brune, and S. Haroche, Rev. Mod. Phys. 73, 565 (2001).

[13] C. Monroe, D. M. Meekhof, B. E. King, W. M. Itano, and D. J. Wineland, Phys. Rev. Lett. 75, 4714 (1995); Q. A. Turchette, C. S. Wood, B. E. King, C. J. Myatt, D. Leibfried, W. M. Itano, C. Monroe, and D. J. Wineland, ibid. 81, 3631 (1998).

[14] L. M. K. Vandersypen, M. Steffen, G. Breyta, C. S. Yannoni, R. Cleve, and I. L. Chuang, Phys. Rev. Lett. 85, 5452 (2000); L. M. K. Vandersypen, M. Steffen, G. Breyta, C. S. Yannoni, M. H. Sherwood, and I. L. Chuang, Nature (London) 414, 883 (2001).

[15] They allowed, for instance, to achieve Bose-Einstein condensation in trapped alkali gases, see M. H. Anderson, J. R. Ensher, M. R. Matthews, C. E. Wieman, and E. A. Cornell, Science 269, 198 (1995); C. C. Bradley, C. A. Sackett, J. J. Tollett, and R. G. Hulet, Phys. Rev. Lett. 75, 1687 (1995); K. B. Daves, M. O. Mewes, M. R. Andrews, N. J. van Druten, D. S. Durfee, D. M. Durn, and W. Ketterle, ibid. 75, 3969 (1995). [16] See, for instance, I. H. Deutsch and P. S. Jessen, Phys. Rev. A
57, 1972 (1998); G. Grynberg and C. Robbilliard, Phys. Rep, 355, 355 (2001), and references therein.

[17] D. Frese, B. Ueberholz, S. Kuhr, W. Alt, D. Schrader, V. Gomer, and D. Meschede, Phys. Rev. Lett. 85, 3777 (2000); N. Schlosser, G. Reymond, I. Protsenko, and P. Grangier, Nature (London) 411, 1024 (2001).

[18] J. Schmiedmayer, Phys. Rev. A 52, R13 (1995); J. D. Weinstein and K. Libbrecht, ibid. 52, 4004 (1995); J. Reichel, W. Hänsel, and T. W. Hänsch, Phys. Rev. Lett. 83, 3398 (1999); N. H. Dekker, C. S. Lee, V. Lorent, J. H. Thywissen, S. P. Smith, M. Drndić, R. M. Westervelt, and M. Prentiss, ibid. 84, 1124 (2000); R. Folman, P. Kruger, D. Cassettari, B. Hessmo, T. Maier, and J. Schmiedmayer, ibid. 84, 4749 (2000).

[19] G. Birkl, F. B. J. Buchkremer, R. Dumke, and W. Ertmer, Opt. Commun. 191, 67 (2001).

[20] F. B. J. Buchkremer, R. Dumke, M. Volk, T. Müther, G. Birkl, and W. Ertmer, Laser Phys. 12, 736 (2002).

[21] R. Dumke, M. Volk, T. Müther, F. B. J. Buchkremer, G. Birkl, and W. Ertmer, Phys. Rev. Lett. 89, 097903 (2002).

[22] D. P. Divicenzo, Fortschr. Phys. 48, 771 (2000).

[23] J. Gruska, Quantum Computing (McGraw-Hill, London, 1999); M. A. Nielsen and I. L. Chuang, Quantum Computation and Quantum Information (Cambridge University Press, Cambridge, 2000).

[24] A. Recati, T. Calarco, P. Zanardi, J. I. Cirac, and P. Zoller, Phys. Rev. A 66, 032309 (2002).

[25] E. Andersson, M. T. Fontenelle, and S. Stenholm, Phys. Rev. A 59, 3841 (1999).

[26] E. Andersson and S. Stenholm, Opt. Commun. 188, 141 (2001).

[27] I. Bouchoule, H. Perrin, A. Kuhn, M. Morinaga, and C. Salomon, Phys. Rev. A 59, R8 (1999); M. Morinaga, I. Bouchoule, J.-C. Karam, and C. Salomon, Phys. Rev. Lett. 83, 4037 (1999).

[28] E. Charron, E. Tiesinga, F. Mies, and C. Williams, Phys. Rev. Lett. 88, 077901 (2002).

[29] A. Peres, Quantum Theory: Concepts and Methods (Kluwer Academic, Dordrecht, 1995).

[30] S. L. Cornish, N. R. Claussen, J. L. Roberts, E. A. Cornell, and C. E. Wieman, Phys. Rev. Lett. 85, 1795 (2000).

[31] An animated illustration of the $\sqrt{\mathrm{SWAP}}$ gate operation can be found at http://www.itp.uni-hannover.de/ $\sim$ eckert/na/index.html

[32] W. Hänsel, J. Reichel, P. Hommelhoff, and T. W. Hänsch, Phys. Rev. A 64, 063607 (2001).

[33] J. Schliemann, J. I. Cirac, M. Kuś, M. Lewenstein, and D. Loss, Phys. Rev. A 64, 022303 (2001).

[34] K. Eckert, J. Schliemann, D. Bruß, and M. Lewenstein, Ann. Phys. (N.Y.) 299, 1 (2002).

[35] R. Paskauskas and L. You, Phys. Rev. A 64, 042310 (2001).

[36] J. R. Gittings and A. J. Fisher, e-print quant-ph/0202051.

[37] $S_{\text {spatial }}$ is calculated by writing $\rho$ as a density matrix in the $\left\{n_{A}, n_{B}\right\}$ occupation number basis. Here $n_{A}, n_{B} \in\{0,1,2\}$ count the number of particles on site $A$ and $B$, respectively. Then $S_{\text {spatial }}=-\operatorname{tr}\left(\rho_{A} \log _{2}\left(\rho_{A}\right)\right)$ where $\rho_{A}$ is the reduced density matrix obtained by tracing out states with $n_{B}=0,1,2$ [36].

[38] P. Zanardi, e-print quant-ph/0104114.

[39] F. B. J. Buchkremer, R. Dumke, H. Levsen, G. Birkl, and W. Ertmer, Phys. Rev. Lett. 85, 3121 (2000). 